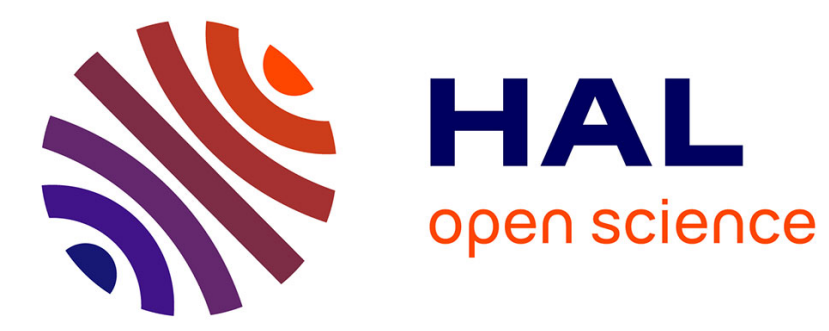

\title{
On Phaser-Based Processing of Impulse Radio UWB over Fiber Systems Employing SOA
}

\author{
H Taki, S Azou, A Hamie, A Al Housseini, A Alaeddine, A Sharaiha
}

\section{To cite this version:}

H Taki, S Azou, A Hamie, A Al Housseini, A Alaeddine, et al.. On Phaser-Based Processing of Impulse Radio UWB over Fiber Systems Employing SOA. Optical Fiber Technology, 2017, 36, pp.3340. 10.1016/j.yofte.2017.02.001 . hal-01456315

\section{HAL Id: hal-01456315 https://hal.science/hal-01456315}

Submitted on 8 Feb 2017

HAL is a multi-disciplinary open access archive for the deposit and dissemination of scientific research documents, whether they are published or not. The documents may come from teaching and research institutions in France or abroad, or from public or private research centers.
L'archive ouverte pluridisciplinaire HAL, est destinée au dépôt et à la diffusion de documents scientifiques de niveau recherche, publiés ou non, émanant des établissements d'enseignement et de recherche français ou étrangers, des laboratoires publics ou privés. 


\title{
On Phaser-Based Processing of Impulse Radio UWB over Fiber Systems Employing SOA
}

\author{
H. Taki*†, S. Azou ${ }^{\dagger}$, A. Hamie ${ }^{* \ddagger}$, A. Al Housseini*, A. Alaeddine* and A. Sharaiha ${ }^{\dagger}$ \\ * Lebanese University, Faculty of Science I, LPE research center, Beirut, Lebanon \\ †ENIB / CNRS UMR 6285 Lab-STICC, Brest, France \\ $\ddagger$ CRITC research center, Arts Sciences and Technology University in Lebanon (AUL)
}

\begin{abstract}
In this study, we adopt a phaser-based processing to enhance the performance of impulse radio over fiber system utilizing SOA. The amplifier has been placed at a distance in the optical link, so as to extend the coverage area of proposed transceiver. Operating in the linear or saturation region for SOA, adds ASE noise or strong nonlinearities acting on the propagated pulses, respectively. Both lead to a degradation in the power efficiency and bit error rate performance. By applying up and down analog chirping technique, we have reduced the ASE power and nonlinearity simultaneously. Based on the 5th Gaussian pulse and Abraha's combination of doublets, a significant improvement has been achieved at extremely low and high input powers entering the amplifier $(<-15 \mathrm{dBm}$ and $0 \mathrm{dBm})$, recording a very good bit error rate performance and power efficiency. Better signal quality was observed after photo-detector, due to the fact that waveforms with lower frequency components are less affected by SOA nonlinearity. Our scheme has proved to be effective for 1 Gbps OOK and 0.5 Gbps PPM transmissions, while reaching a distance of $160 \mathrm{~km}$ in the optical fiber.
\end{abstract}

\section{INTRODUCTION}

The raising demand of high quality data transfer necessitates a periodic advancement in the communication system technologies. Numerous applications, like sensor networks, local and wide area networks, remote sensing and radar, require a high speed transmission within a short range coverage areas [1]. The most suitable technique fitting this request is Ultra Wide Band (UWB), based on transmitting signals with an extremely large bandwidth and small radiated power. A lot of features make UWB an attractive technology, mainly the high data speed, flexibility in reconfiguring bit rate and power, immunity to multipath propagation, accurate mobile user location, very good time domain resolution, besides to easy data protection [2] [3]. The first global standardization for UWB was done in 2002, when it has been authorized by the Federal Communication Commission (FCC) to use the $[3.1,10.6] \mathrm{GHz}$ band, where the emitted power must stay below $-41.3 \mathrm{dBm} / \mathrm{MHz}$ in order to protect existing wireless services [4]. A particular application of UWB is the Impulse Radio (IR), relying on transmitting ultra short pulses directly in baseband, interesting features of this technique being the low cost and complexity required [5]-[7]. UWB free space propagation is typically limited at a few meters due to the FCC power constraints, hence widening the service area via optical links is an attractive solution that has been recently demonstrated [8]-[12]. Over fiber transmission is either based on direct optical pulse generation [13], [14], or electrical gen- eration followed by intensity modulation of UWB signals [15]. Adopting an in-line amplifier in the optical access network is a key approach for getting a reach extension. In our study, we investigate the usage of Semiconductor Optical Amplifier (SOA) [16] to perform this function, as it is a low cost optical component which is pertinent to be employed with simple impulse radio schemes. However, such a technique may lead to some drawbacks, mainly the Amplified Spontaneous Emission (ASE) noise produced [17], [18] and the nonlinear effects acting on the pulse waveform [19]. Both degrade the performance of the optical system, lower power efficiencies being achieved due to distortion, besides to the increased number of errors obtained at receiver side. In order to overcome the SOA distortions, noise reduction and linearization techniques have to be applied, without adding more complexity to the conventional system architecture. Various methodologies concerning these two topics have been investigated in literature [20]-[28]. The objective in [19] was to pre-distort against SOA nonlinear impairments by adjusting the parameters of input waveforms. This scheme was based on global optimization requiring a feedback in the electro-optical system. A special effort is applied here to examine the ability of chirping in upgrading the performance, by the use of phaser-based processing we have overcome SOA nonlinearities and ASE noise simultaneously. Following the work of Nikfal et al. [29], who has recently succeeded in enhancing the signal-to-noise ratio of UWB systems via group delay phasers, symmetrical up and down chirping has shown to be effective against ASE influence as a first step. Then we proved that SOA nonlinear effects could be also mitigated, as frequency decomposition reduces the order of four wave mixing and cross gain modulation against propagated signals. Up-down chirping could be considered as a pre-post distortion technique, which has improved the power efficiency of transmitted impulse radio, while traveling along a broad distance in the optical link, thanks to chirping.

\section{IR-UWB OVER FIBER SYSTEM ARCHITECTURE AND SOA IMPACT ON OUTPUT PULSES RESPONSE}

The Radio-over-Fiber (RoF) utilized is depicted in fig. 1. The transmitter relies on a single arm Mach-Zehnder Modulator (MZM) with half-wave voltage $V \pi$ of $6 \mathrm{~V}$, biased at $V_{D C}=1.5 V_{\pi}$ by the electrical IR-UWB signal (with a peakto-peak voltage $V_{p p}$ ), a continuous wave light being applied at its input (laser diode signal at $1540 \mathrm{~nm}$ wavelength). The 
channel is made of a Single Mode Fiber (SMF) and an inline amplifier (SOA) for the reach extension purpose, with an injected bias current $I_{\text {bias }}$. At the receiver side (just before

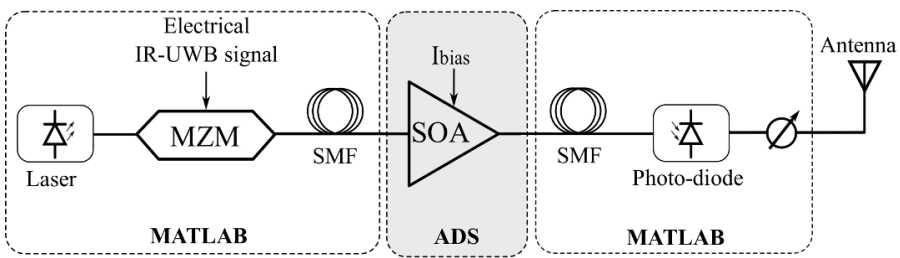

Fig. 1: Block diagram of the Impulse Radio over fiber system with Matlab/ADS co-simulation.

wireless transmission), a photo-detector (assumed to be noise free) converts the optical power into an electrical voltage signal to be eventually attenuated before entering the antenna (so as to meet FCC mask). The same ADS software-based model as in [30] is adopted for the SOA (INPHENIX-IPSAD1501), with unchanged physical properties, as it has proved to be highly accurate and in very good agreement with experimental data. Increasing the transmission distance requires operating in the saturated region of the SOA, which yields various nonlinear impairments affecting the transmitted signal. On the non-coherent receiver side, the resulting distortion will be characterized in terms of power efficiency with respect to the FCC mask, defined as:

$$
\eta=\frac{\int_{3.1 \mathrm{GHz}}^{10.6 \mathrm{GHz}} S_{p}(f) d f}{\int_{3.1 \mathrm{GHz}}^{10.6 \mathrm{GHz}} S_{F C C} d f} \times 100 \%
$$

where $S_{p}(f)$ denotes the power spectral density (PSD) for a pulse $p(t)$ [31]. $\eta$ is computed after photo-detector while considering antenna effects are ignored. To have a look at SOA impact, we have studied the spectral response of the 5-th order derivative Gaussian pulse before and after being passed through SOA, with a peak-to-peak voltage fixed at $0.5 \mathrm{~V} \pi$, and the amplifier biased at $250 \mathrm{~mA}$. Figure 2 shows the spectrum in electrical domain (solid-blue), where the dot-dashed-green and dashed-red curves correspond to the spectra obtained at SOA output, while operating in the linear region $(-25 \mathrm{dBm}$ input power) and saturation region ( $0 \mathrm{dBm}$ input power $)$, respectively. In both cases, the PSD of the optical pulse violates FCC mask in the most restricted band corresponding to GPS $[0.96-1.61] \mathrm{GHz}$. At linear region, the distortion in the spectrum is due to the noise flat spectral response; while at saturation, it is due to the strong nonlinearities acting on the pulse waveform. Hence, the average signal power has to be reduced so as to meet the regular limit, leading to a significant impact on the power efficiency (degradation $>$ $35 \%$ ) at both operating points, specially for the noisy case. In our recent work [19], we have studied the power efficiency versus SOA input power while considering several peak-topeak voltages. It has been shown that high input powers bring strong nonlinearities specially at large $V_{p p}$, and low powers are associated with a small signal-to-noise (SNR) ratio particularly at low $V_{p p}$. We point out also that the compromise between

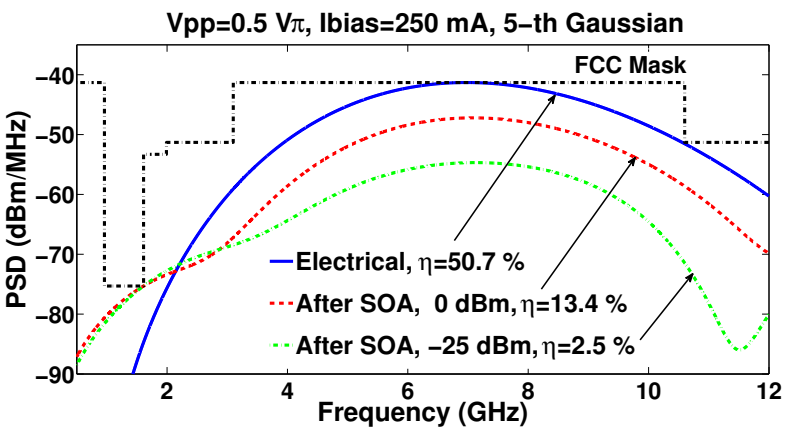

Fig. 2: Power Spectral Density of $5^{\text {th }}$ Gaussian in electrical domain and after SOA (while operating in the linear or saturation region).

linearity and SNR is controlled by the maximum voltage swing. The latter has been fixed at $V_{p p}=0.5 V \pi$ when studying the nonlinearity aspect in the remaining part of the paper, as it is a medium value that well describes the SOA behavior versus operating point.

\section{PRINCIPLE OF A TRANSMISSION SYSTEM UTILIZING UP AND DOWN CHIRPING TECHNIQUE}

The system architecture described in fig. 3 has been investigated by Nikfal [29], with the aim of enhancing the SNR at receiver side. The key function is performed by the phaser, which has a dispersive structure with controllable group delay [32]. Such a component applies linear up or down chirping for the input signal, thus stretching or compressing the pulse without making a change in the total energy. Therefore, if the ratio of the pulse duration before to after phaser is $M$, then the corresponding value for the average power is $1 / M$. The transmitter operates a linear up chirping for the data pulses before entering the channel, while at receiver side, the instantaneous frequencies are equalized so as to compress the pulse to an enhanced waveform that is essentially identical to the original one (down chirping being performed with an absolutely equal slope to that of the transmitter). Several investigations originating from the chirp concept have been reported in literature, which include real-time spectrum sniffing and enhancing the resolution of analog signal-processing systems [33], [34]. Equations (2), (3), and (4) describe the time and frequency domain expressions of the chirped signal, besides to the frequency dependent time delay function $\tau(f)$, where $x(t)$ is the original pulse before entering the phaser, $a$ is the chirping slope, and $b$ is the delay offset. Figure 4 (left) shows the time waveform of an up-chirped $5 t h$ order Gaussian pulse, with a chirping slope of $0.0472 \mathrm{~ns} / \mathrm{GHz}$; when we apply a down chirping with opposite polarity, the resultant pulse can exactly match the original as verified in fig. 4 (right). A key point to mention is that no local oscillator has been used to generate the chirped pulse, it is a simple phaser which follows the pulse shaper. Figure 5 (left) states the Wigner Ville Distribution [35] of the normal pulse; as can be seen, several frequency components exist at different time instants simultaneously, specially at the center of the waveform. By up chirping we make a partial separation so that the peak 
frequency increases with time, less frequency overlap being obtained for the chirped pulse as illustrated in fig. 5 (right). In the case of up-chirping, we consider $b=0$ as no need for a delay offset, whereas for down chirping, the value of $b$ must not go under the maximum time shift applied in the negative sense. Chirping affects only the phase of the spectrum without varying its magnitude (4), hence there is no influence on the power spectral density, which is a positive point for this approach, as no drawback is observed in terms of power efficiency.

$$
\begin{gathered}
y(t)=x(t+\tau(f)) \\
\tau(f)=a f+b \\
Y(f)=X(f) \exp (2 \pi f \tau(f) j) \\
=X(f) \exp \left(2 \pi a f^{2} j\right) \exp (2 \pi f b j)
\end{gathered}
$$

Table I states different values of chirping slopes, each associated with a maximum time shift $\tau_{\max }$, computed as $\tau_{\max }=$ $a \times f_{\max }=a \times 10.6 \mathrm{GHz}$, as the energy of input pulses is negligible for frequencies above the FCC upper bound. Assuming $f_{\max }$ exists at the end of the pulse interval, the new pulse duration $T_{p}$ becomes the old one $(0.5 \mathrm{~ns})$ added to $\tau_{\max }$. Therefore, the data rate for an On Off Keying (OOK) modulated signal having a unit processing gain is $1 / T_{p}$, whereas for a non-coherent Pulse Position Modulation (PPM) it is $1 /\left(2 T_{p}\right)$. In [29], they have proved an enhancement

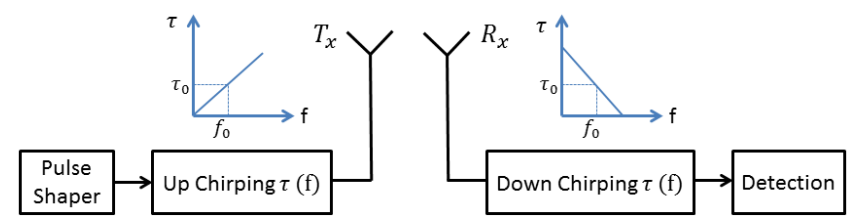

Fig. 3: The principle of the proposed transmission system utilizing up and down chirping.
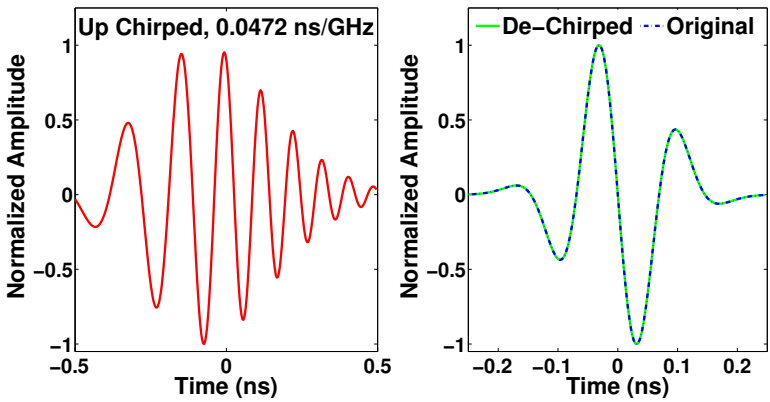

Fig. 4: The time waveforms of the 5th up-chirped Gaussian with a duration of $1 \mathrm{~ns}$ (left), besides to the down-chirped pulse in comparison to the original one with a duration of $0.5 \mathrm{~ns}$ (right).

in the SNR; unfortunately, there was no evaluation in terms of bit error rate (BER) performance. Here we measure the BER versus $E b / N_{0}$ in an AWGN channel, assuming that the noise power depends on the transmitted signal. We can see an improvement for both OOK and PPM modulations as
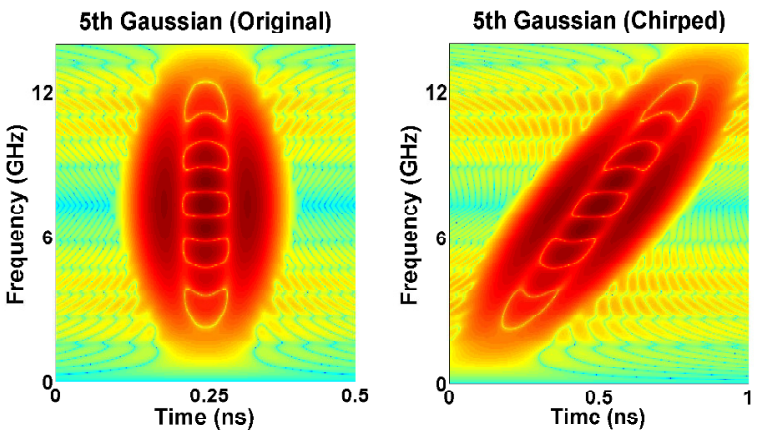

Fig. 5: The Wigner Ville Distribution of $5 t h$ original Gaussian (left) besides to the up-chirped pulse (right) in electrical domain, where the chirping slope is $0.0472 \mathrm{~ns} / \mathrm{GHz}$.

\begin{tabular}{|c|c|c|c|c|}
\hline $\begin{array}{c}\text { Chirping Slope } \\
\text { (ns/GHz) }\end{array}$ & $\begin{array}{c}\tau_{\max } \\
(\mathrm{ns})\end{array}$ & $\begin{array}{c}T_{p} \\
(\mathrm{~ns})\end{array}$ & $\begin{array}{c}R_{O O K} \\
(\mathrm{Gbps})\end{array}$ & $\begin{array}{c}R_{P P M} \\
(\mathrm{Gbps})\end{array}$ \\
\hline 0 & 0 & 0.5 & 2 & 1 \\
\hline 0.0052 & 0.055 & 0.555 & 1.8 & 0.9 \\
\hline 0.0118 & 0.125 & 0.625 & 1.6 & 0.8 \\
\hline 0.0202 & 0.214 & 0.714 & 1.4 & 0.7 \\
\hline 0.0314 & 0.333 & 0.833 & 1.2 & 0.6 \\
\hline 0.0472 & 0.5 & 1 & 1 & 0.5 \\
\hline
\end{tabular}

TABLE I: The set of chirping slopes dependent in the study, and the corresponding time shift, besides to the pulse duration and resultant data rate for OOK or PPM.

observed in fig. 6, where the chirping slope is $0.0472 \mathrm{~ns} / \mathrm{GHz}$. Lower number of errors is obtained at the cost of data rate as illustrated in fig. 7, where OOK operates at the maximum data rate and PPM occupies only the half. Increasing the chirping slope broadens the pulse duration, and consequently degrades the transmission speed but enhances the quality of service on the other side.

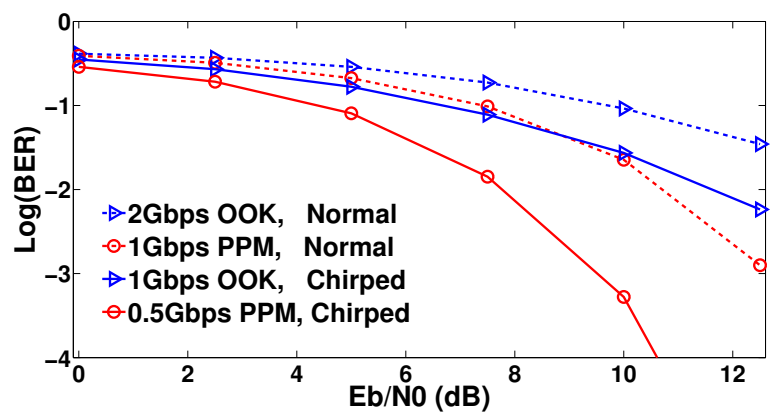

Fig. 6: Bit error rate versus $E b / N_{0}$ for normal and chirped (with a slope of $0.0472 \mathrm{~ns} / \mathrm{GHz}$ ) transmissions over AWGN channel, while considering OOK and PPM modulation formats.

\section{The BENEFITS OF ChIRPING IN A SOA-BASED IR-UWB OVER FIBER SYSTEM}

The great potential offered by up and down chirping technique presented in the previous section, has motivated us to go towards SOA-based IR-UWB over fiber systems, which may 


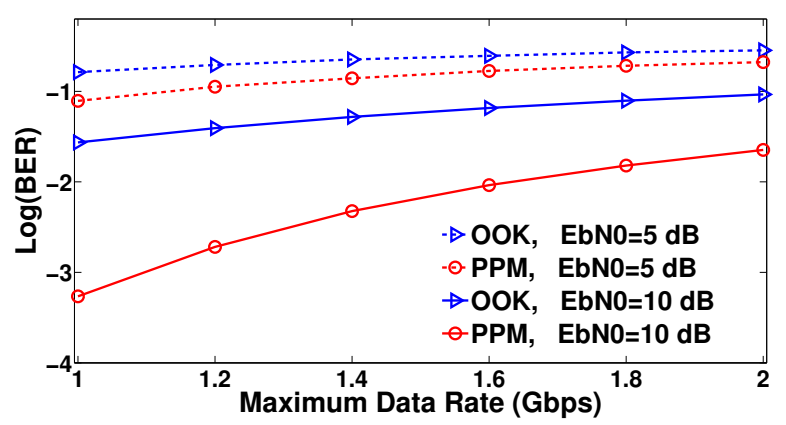

Fig. 7: Bit error rate in an AWGN channel versus the maximum data rate could be achieved (associated with a specific chirping slope) for OOK and PPM modulation formats.

suffer from ASE effects. Noise reduction in communication systems is a general topic that has been investigated in numerous studies. Here we adopt the chirping mechanism for attenuating the noise, as it is a low cost analog approach. The block diagram of the proposed system is described in fig. 8, with up and down chirping taking place before and after the optical system respectively. The initial target was to follow the work of [29] and examine the performance considering a radio over fiber application. Then, as will be shown in the sequel, the chirping process is also capable to improve the power efficiency of amplified pulses by reducing the order of SOA nonlinearity. To the knowledge of the authors, this has not been examined in the literature until now.

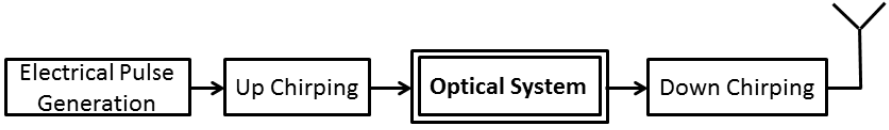

Fig. 8: Up and down chirping applied before and after the optical system respectively, which includes the MZM, fiber, SOA in-line amplifier, and photo-detector.

\section{A. Reducing the impact of ASE noise via chirping}

The Gaussian and burst noises have been considered in [29], here we prove that even for ASE noise a notable increase in the SNR could also be achieved. Figure 9 shows the time waveforms of the 5 th order Gaussian pulse obtained at SOA output, with (right) and without (left) chirping, where the slope is $0.0472 \mathrm{~ns} / \mathrm{GHz}$ and $\mathrm{SOA}$ operates in the linear region $(-20$ $\mathrm{dBm}$ input power). Like in AWGN channel, the reduction in the noise impact translates into a better BER performance, as illustrated in fig. 10 for several bit rates of OOK and PPM modulation formats, where $V_{p p}=0.1 V \pi$ and $I_{\text {bias }}=250$ $\mathrm{mA}$.

\section{B. SOA linearization via chirping}

Besides to the improvement in system performance while operating in the SOA linear region, we can also enhance the power efficiency of pulses obtained at SOA output, by reducing the order of nonlinearity through up and down
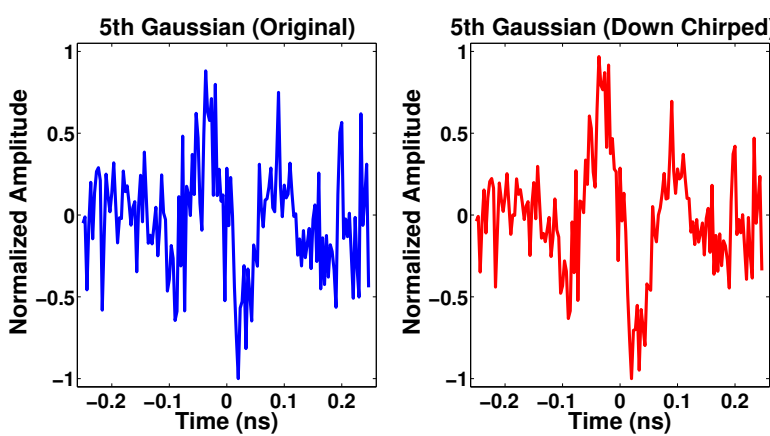

Fig. 9: The time waveforms of the noisy 5th Gaussian obtained at SOA output, with (right) and without (left) chirping, where $\mathrm{P}-\mathrm{SOA}=-20 \mathrm{dBm}, V_{p p}=0.1 \mathrm{~V} \pi$, and $I_{\text {bias }}=250 \mathrm{~mA}$.

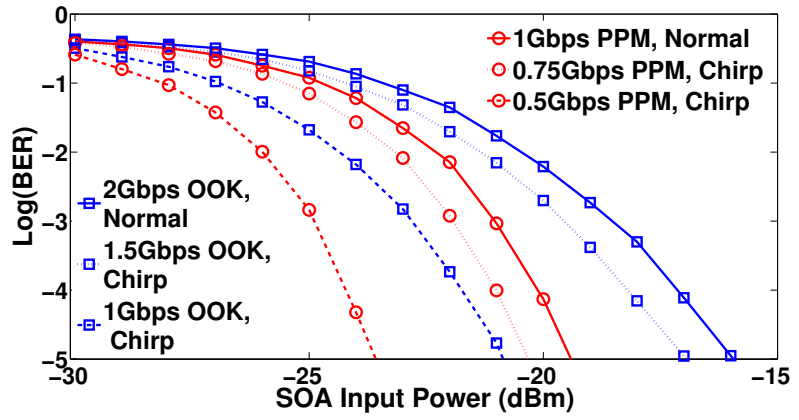

Fig. 10: Bit error rate versus SOA input power for several data rates of normal and chirped transmissions, considering OOK and PPM modulation formats, where $V_{p p}=0.1 V \pi$ and $I_{\text {bias }}=250 \mathrm{~mA}$.

chirping. A lower order of cross gain modulation and fourwave mixing is obtained if a single frequency is processed at a time, rather than amplifying the whole frequency components simultaneously [16]. Hence, stretching the pulse over time reduces the SOA nonlinearity, as different frequency components can pass through the amplifier at separate time instants. Based on this concept, we enter chirped signals at the SOA input in order to get less distortion, and after photodetection the original pulse can be reconstructed via down chirping. This strategy could be classified as a new type of linearization in addition to existing pre-distortion and postdistortion schemes employed for power amplifiers [20]-[27]. The average cross-correlation between pulses before and after SOA has been studied for different combinations of sinusoids, where the total set includes 4 frequencies, $\left(f_{1}=4 \mathrm{GHz}, f_{2}=6\right.$ $\mathrm{GHz}, f_{3}=8 \mathrm{GHz}, f_{4}=10 \mathrm{GHz}$ ). Figure 11 reveals that a better correlation between electrical and optical pulses is achieved for combinations based on a lower number of frequencies.

$$
\begin{gathered}
x_{5}(t)=\frac{A}{\sqrt{2 \pi}}\left(\frac{-t^{5}}{\sigma^{11}}+\frac{10 t^{3}}{\sigma^{9}}-\frac{15 t}{\sigma^{7}}\right) \exp \left(-\frac{t^{2}}{2 \sigma^{2}}\right) \\
C_{D}(t)=a_{1} x_{21}(t)+a_{2} x_{22}(t-\tau) \\
x_{2 i}(t)=\frac{A}{\sqrt{2 \pi} \sigma_{i}^{3}}\left(k \frac{t^{2}}{\sigma_{i}^{2}}-1\right) \exp \left(-\frac{t^{2}}{2 \sigma_{i}^{2}}\right)
\end{gathered}
$$

The high power efficiency offered by the 5 th derivative Gaussian pulse, besides the acceptable order of generation 


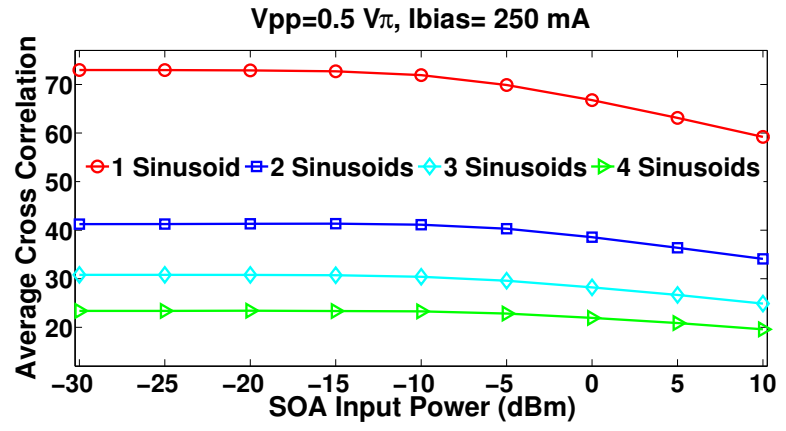

Fig. 11: Average cross correlation between electrical and optical (after photo-detector) waveforms, versus SOA input power for several combinations of sinusoids, $V p p=0.5 \mathrm{~V} \pi, I_{\text {bias }}=250 \mathrm{~mA}$.

complexity, both make it an attractive target for impulse radio system designers [36]. In addition to Abraha's pulse developed recently, based on a linear combination of doublets [31], which exhibits a better power efficiency with much lower order of complexity, these two waveforms will be the candidates in our work. Equations (5), (6), and (7) describe the time expressions of utilized pulses, besides to the Gaussian doublet, where $a_{1}$ and $a_{2}$ are the weighting coefficients, $\sigma$ is the pulse shaping factor, $\tau$ is a time delay between pulses fixed at $\tau=25 \mathrm{ps,}$ and $k=1.16$ is an arbitrary scaling for the modified doublet. The parameters of Abraha's combination are set as $a_{1}=-a_{2}$ and $\sigma_{1}=\sigma_{2}$, in order to perform a high pass filtering by the frequency oscillating term resulted from time shifting. To examine first the potential of chirping, several slopes have been considered for both waveforms. Figure 12 illustrates

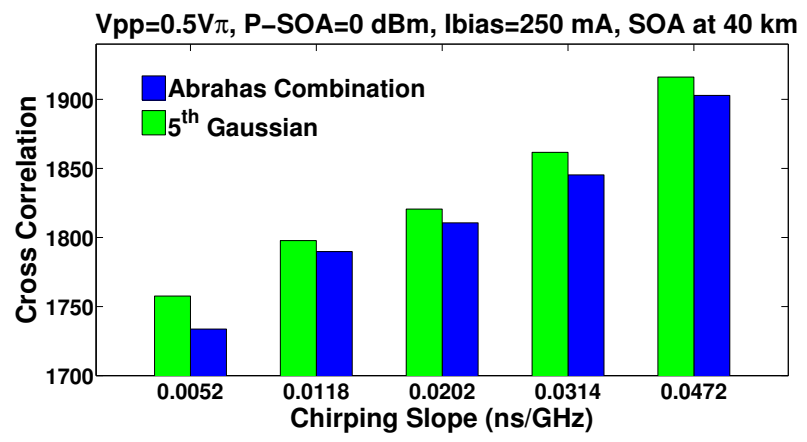

Fig. 12: Cross Correlation between electrical and optical waveforms versus chirping slope, for the 5th Gaussian pulse and Abraha's combination, where $V p p=0.5 V \pi, I_{\text {bias }}=250 \mathrm{~mA}, \mathrm{P}-\mathrm{SOA}=0 \mathrm{dBm}$.

that a better cross correlation is being achieved at higher chirping slopes, while operating at a strong saturation point $(0 \mathrm{dBm})$, promising with an enhancement in the pulse power efficiency when chirping is adopted. The dominance of the 5 th Gaussian over $C_{D}$ is due to original pulse dynamics. The highest slope $(0.0472 \mathrm{~ns} / \mathrm{GHz})$ has been considered in the remaining part of the study. For a better clarification, we can see in fig. 13 the input-to-output amplitude mapping associated to Abraha's combination without (left) and with (right) chirping, the distribution of the points is more close to the linear response, that validates the effectiveness of our approach.

\section{Bias current influence}

Figure 14 plots the power efficiency versus biasing current for the normal and chirped pulses, where $\mathrm{P}-\mathrm{SOA}=0 \mathrm{dBm}$ and $V_{p p}=0.5 V \pi$. Abraha's waveform is more sensitive to SOA nonlinear impairments, as the spectrum in electrical domain has a high $10 \mathrm{~dB}$ bandwidth, so any spectral deviation is enough to violate FCC mask, and a fast step-down in the efficiency will be obtained. For $I_{\text {bias }}=100 \mathrm{~mA}$, only a small decrease in the power efficiency is noticed for the 5th Gaussian with respect to the electrical domain response (table II), unlike the combination of doublets where a significant degradation (close to $38 \%$ ) is observed. Operating at larger biasing currents brings strong nonlinearities acting on the propagated pulses, and consequently lower power efficiencies are being obtained for both waveforms. After $200 \mathrm{~mA}$, only a small change appears, as the amplifier reaches a saturated gain due to the high input power and current applied. The key point here is that we can always see an improvement for the chirped signals over original ones. The biasing current will be fixed at $250 \mathrm{~mA}$ for next simulations, as associated with the highest optical gain matching the request of reach extension.
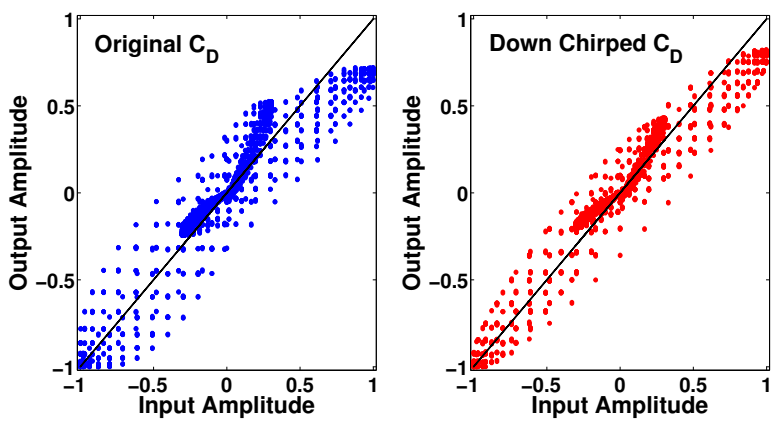

Fig. 13: The input-to-output mapping for Abraha's combination after being passed through SOA, without (left) and with (right) chirping, where P-SOA $=0 \mathrm{dBm}, I_{\text {bias }}=250 \mathrm{~mA}, V_{p p}=0.5 \mathrm{~V} \pi$.

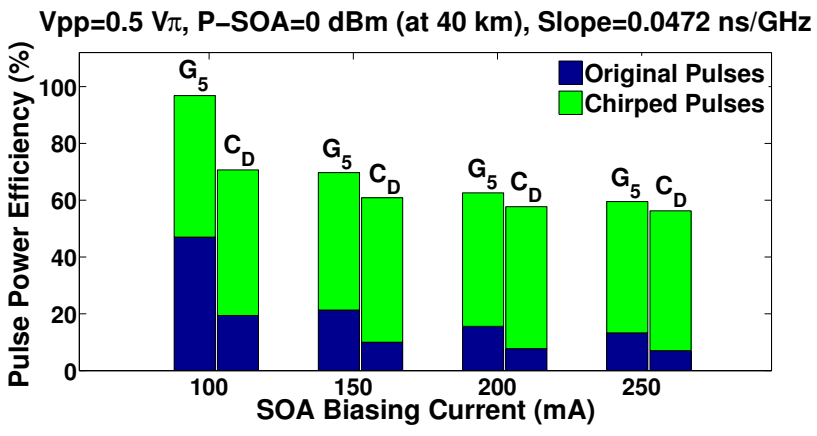

Fig. 14: Power Efficiency of original and chirped pulses directly after SOA (at $40 \mathrm{~km}$ ) for several biasing currents, where $V p p=0.5 V \pi$ and $\mathrm{P}-\mathrm{SOA}=0 \mathrm{dBm}$. 


\begin{tabular}{|c|c|c|c|c|c|c|c|}
\hline Pulses & $\sigma(\mathrm{ps})$ & $\eta(\boldsymbol{\%})$ Elec & $f_{\text {peak }}(\mathrm{GHz})$ & $B_{3}(\mathrm{GHz})$ & $B_{10}(\mathrm{GHz})$ & $B L_{34}(\mathrm{GHz})$ & $\eta(\boldsymbol{\%}) \mathrm{SOA}$ \\
\hline Original 5th Gaussian & 51 & 50.7 & 7 & 3.43 & 6.17 & 6.11 & 13.7 \\
\hline Chirped 5th Gaussian & 51 & 50.7 & 7.04 & 3.63 & 6.63 & 4.87 & 46.24 \\
\hline Original Abraha's Pulse & 43.02 & 57.2 & 6.45 & 3.82 & 6.84 & 6.05 & 6.96 \\
\hline Chirped Abraha's Pulse & 43.02 & 57.2 & 6.63 & 4.14 & 7.43 & 4.93 & 49.23 \\
\hline
\end{tabular}

TABLE II: Pulse Shaping Factors of electrical generated waveforms, and spectral characteristics $\left(f_{\text {peak }}, B_{3}, B_{10}, B L_{34}\right)$ of corresponding pulses at SOA output (placed at $40 \mathrm{~km}$ ). Besides to the power efficiencies (\%) in electrical domain and after SOA, with $P_{S O A}=0 d B m$, $I_{\text {bias }}=250 \mathrm{~mA}$, and $V_{p p}=0.5 V_{\pi}$.

\section{Time waveforms and corresponding spectra of the pulses with and without chirping}

Figures 15 and 16 describe the time and frequency plots of our pulses in electrical domain and after SOA (at the photodetector output), where the spectra are plotted in the noisefree case (ASE noise not considered for spectrum readability). Table II states the parameters of applied waveforms, besides to the power efficiencies and spectral characteristics evaluated at SOA output. $B L_{34}$ is defined as the left $-34 \mathrm{~dB}$ bandwidth (distance between the lower frequency $f_{L}$ and $f_{\text {peak }}$ ), this criterion is useful to evaluate the spectrum in correspondence with GPS band [19]. As shown, the same waveforms are generated in electrical domain, and chirping has no drawback on the pulse spectral behavior. A significant raise in the power efficiency is observed for the two waveforms, specially for Abraha's combination, where we have an increase in the $3 \mathrm{~dB}$ and $10 \mathrm{~dB}$ bandwidth from, 3.82 to $4.14 \mathrm{GHz}$ and 6.84 to 7.43 $\mathrm{GHz}$ respectively, besides to the $1.12 \mathrm{GHz}$ reduction in $B L_{34}$, both permit for a step up in the PSD under FCC mask while covering a large spectral area without violating the regular limit, even in the most power restricted region corresponding to GPS band. The spectrum is centered at $f_{\text {peak }}=6.63 \mathrm{GHz}$, which is not so far from the middle of UWB mask $(6.85 \mathrm{GHz})$. A lower power efficiency is recorded for the chirped 5th Gaussian with respect to Abraha's pulse after SOA, knowing that it has a higher cross correlation for the same conditions (fig. 12). This is due to the initial difference in the efficiency of electrical waveforms; otherwise, the optical-to-electrical ratio of the 5th Gaussian $(46.24 \% / 50.7 \%=0.91)$ is greater than for Abraha's combination $(49.23 \% / 57.2 \%=0.86)$. An important point to mention is that, unlike usual linearization techniques, our approach is based on complementary pre and post-distortion being performed symmetrically on the transmitter and receiver sides.

\section{Case of a Modulated Impulse Radio Signal PROPAGATING OVER OPTICAL FiBER LINK}

In this section, we consider a modulated impulse radio signal passing through an optical channel, while operating at strong saturation $(\mathrm{P}-\mathrm{SOA}=0 \mathrm{dBm})$ with a large biasing current $\left(I_{\text {bias }}=250 \mathrm{~mA}\right)$, where $V_{p p}=0.5 \mathrm{~V} \pi$ and SOA placed $40 \mathrm{~km}$ apart from MZM. Certain factors affect the time and spectral properties of a signal propagating over optical link, mainly the covered distance, peak-to-peak power swing,
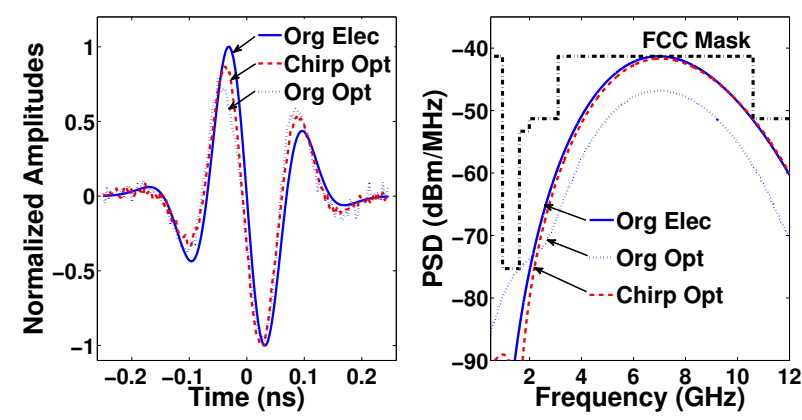

Fig. 15: Original (Org) 5th Gaussian in electrical domain (Elec) and at SOA output (Opt), besides to the chirped pulse after SOA, where the chirping slope is $0.0472 \mathrm{~ns} / \mathrm{GHz}$.
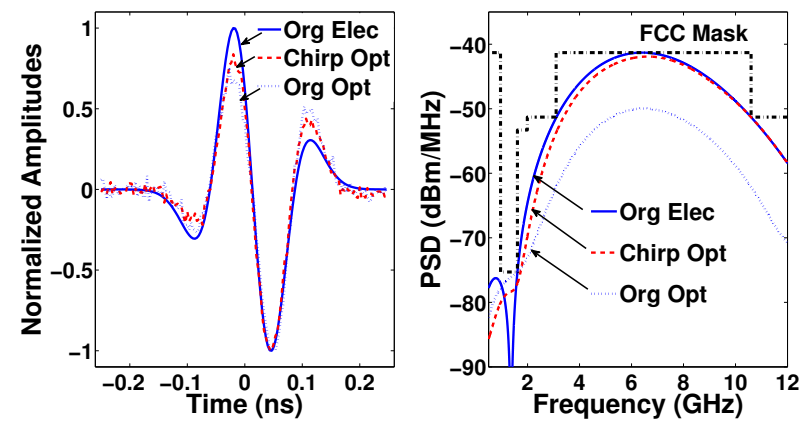

Fig. 16: Original (Org) Abraha's combination in electrical domain (Elec) and at SOA output (Opt), besides to the chirped pulse after SOA, where the chirping slope is $0.0472 \mathrm{~ns} / \mathrm{GHz}$.

fiber input power, and pulse dynamics. The two pieces of SMF (before and after SOA) have an attenuation factor of $0.2 \mathrm{~dB} / \mathrm{Km}$, with a chromatic dispersion of $17 \mathrm{ps} / \mathrm{nm} / \mathrm{km}$. In order to evaluate our scheme taking into account a stream of modulated pulses, a new criterion of power efficiency has been defined as

$$
\eta_{M}=\frac{P_{\mathcal{F}}}{\max \left(\overline{P_{\mathcal{F}}}\right)}
$$

where $P_{\mathcal{F}}$ stands for the power collected over a frequency band of interest $\mathcal{F}$ (typically the [3.1-10.6] $\mathrm{GHz}$ band) for the electrical signal at antenna input, and $\max \left(\overline{P_{\mathcal{F}}}\right)$ denoting the total power evaluated over the same band for an OOK or PPM modulated signal based on the sinc pulse, which is optimal in the sense that it corresponds to a $100 \%$ spectrum use (full coverage of the spectral mask). In fig. 17 we can 


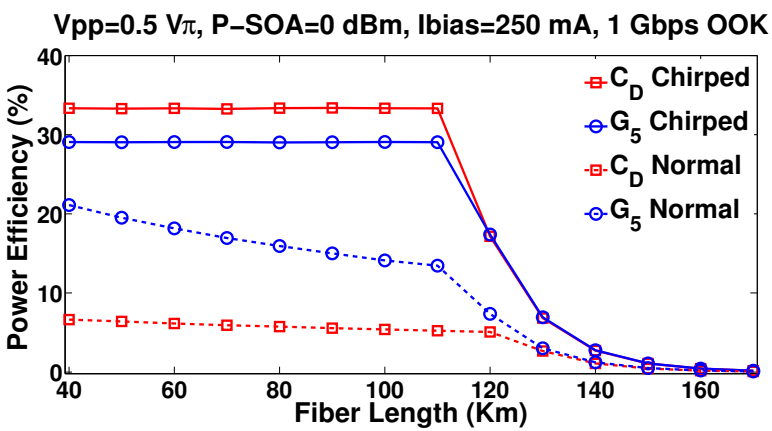

Fig. 17: Power Efficiency versus fiber length for $1 \mathrm{Gbps}$ OOK based on the utilized pulses, P-SOA=0 dBm, $I_{\text {bias }}=250 \mathrm{~mA}$, $V_{p p}=0.5 \mathrm{~V} \pi$, where $\mathrm{SOA}$ is placed at $40 \mathrm{~km}$.
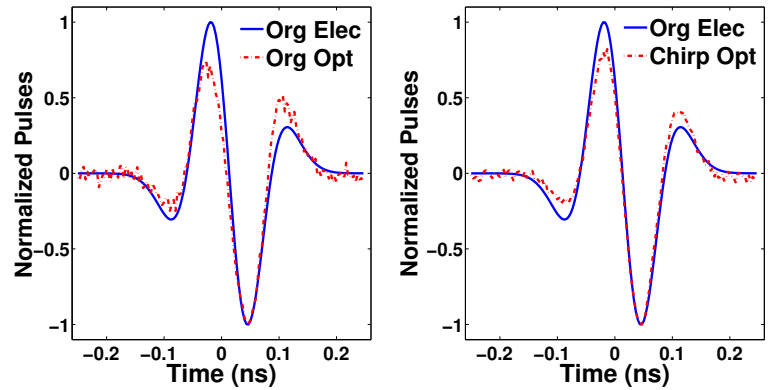

Fig. 18: Abraha's combination at $100 \mathrm{~km}(60 \mathrm{~km}$ after SOA) with (right) and without (left) chirping, where P-SOA $=0 \mathrm{dBm}$, $I_{\text {bias }}=250 \mathrm{~mA}, V_{p p}=0.5 \mathrm{~V} \pi$.

see the advantage of chirping technique considering a $1 \mathrm{Gbps}$ OOK; for both waveforms the chirped signal outperforms the original one while traveling along a large enough distance, knowing that no pre-amplifier has been placed for modulated signals before the antenna. This improvement is justified by the fact that the envelope of spectral spikes for OOK or PPM takes the shape of basic transmitted waveform, so perfect pulse shapes lead to an efficient spectrum associated with the modulated impulse radio. Due to frequency shaping and deviation caused in fiber [10], the power efficiency decreases as the signal propagates over the optical channel, since the central frequency and $B_{10}$ are no more preserved specially at large extension link. The flat response of the curves between $40 \mathrm{~km}$ and $110 \mathrm{~km}$, is due to the fact that all spectra are already above FCC mask, therefore the signal has to be systematically attenuated so as to respect the standard limit. At large distances the spectrum goes under FCC mask; hence no need for up and down chirping, as nonlinearity is not harmful for free spectra below the regular mask. The time waveforms of Abraha's combination at $100 \mathrm{~km}$ has been plotted in fig. 18; as we can see, the de-chirped pulse has a better correlation with the electrical waveform than the original one, where the small intensity of the noise observed is due to SOA strong saturation. The PSD of the OOK signal based on $C_{D}$ at SOA output is plotted in fig. 19. Evidently, the spectral shaping at low frequency band is the reason beyond efficiency enhancement, so a higher power level can be achieved by the spectra when
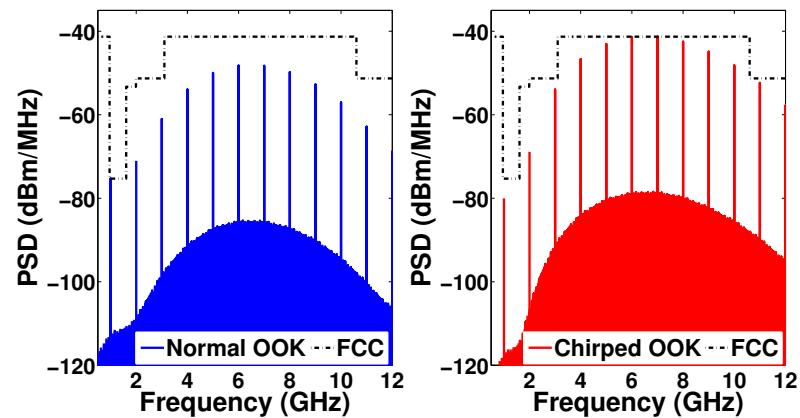

Fig. 19: PSD of 1 Gbps OOK based on original (left) and chirped (right) Abraha's combination after SOA (at $40 \mathrm{~km}$ ), where P-SOA=0 $\mathrm{dBm}, I_{\text {bias }}=250 \mathrm{~mA}, V_{p p}=0.5 \mathrm{~V} \pi$.
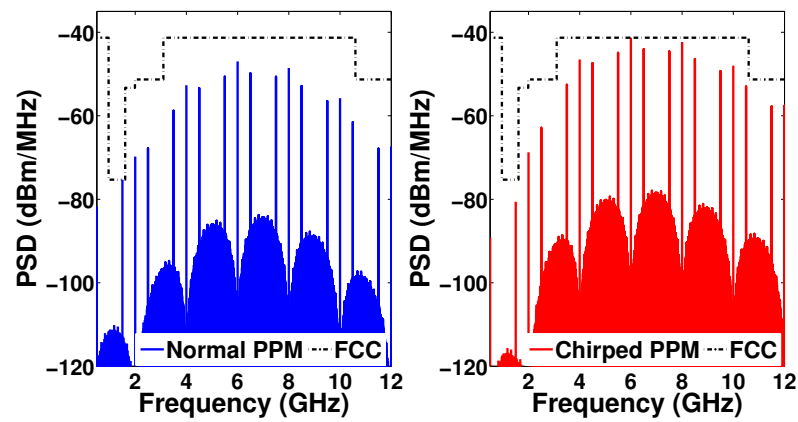

Fig. 20: PSD of 0.5 Gbps PPM based on original (left) and chirped (right) Abraha's combination after SOA (at $40 \mathrm{~km}$ ), where P-SOA=0 $\mathrm{dBm}, I_{\text {bias }}=250 \mathrm{~mA}, V_{p p}=0.5 \mathrm{~V} \pi$.

adapted to FCC mask, as also shown in fig. 20 for the case of 0.5 Gbps PPM. Figure 21 informs that the same conclusion could be drawn for PPM; hence, the proposed up-down chirping technique has proved to be effective while reaching a coverage of $160 \mathrm{~km}$, which can fit the demand of Fiber-tothe-Home (FTTH) networks [37]. Here we consider OOK and PPM formats with non-coherent energy detection; otherwise, chirping is a general approach which could be adopted with other modulation schemes. The performance gain is due to linearizing the SOA characteristics, which is theoretically not limited to any type of transmission. A number of features could be added for this work in comparison to our recent publications [19] regarding pre-distortion. In fact, here there is no need for a feedback in the electro-optical system to apply an optimization process, saving thus complexity and computational time. Since with chirping, the improvement in the efficiency is relative to the slope of the phaser, accordingly it is a well known relation and not a search for the optimal settings. Besides, the new scheme performs well for both 5th Gaussian and Abrahas combination, unlike the previous approach which is more effective when adopted with combinations of waveforms.

\section{CONCLUSION}

In this paper, an analog up-down chirping technique has been applied for the first time in SOA-based impulse radio over fiber system. The aim is to enhance the performance 
$\mathrm{Vpp}=0.5 \mathrm{~V} \pi, \mathrm{P}-\mathrm{SOA}=0 \mathrm{dBm}$, lbias $=250 \mathrm{~mA}, 0.5 \mathrm{Gbps} \mathrm{PPM}$

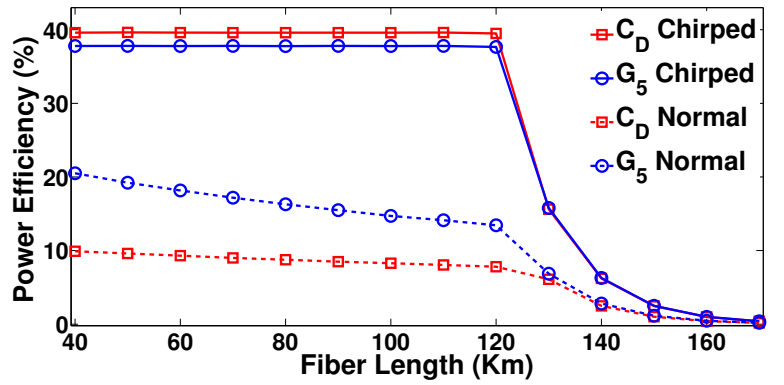

Fig. 21: Power Efficiency versus fiber length for $0.5 \mathrm{Gbps}$ PPM based on the utilized pulses, P-SOA $=0 \mathrm{dBm}, I_{\text {bias }}=250 \mathrm{~mA}$, $V_{p p}=0.5 \mathrm{~V} \pi$, where SOA is placed at $40 \mathrm{~km}$.

in terms of bit error rate and power efficiency. Utilizing phaser-based processing, we mitigated the order of SOA nonlinearity at strong saturation, besides to the ASE noise reduction at linear region. Relying on a realistic SOA physical model, a significant increase in the pulse efficiency of the 5th Gaussian $(>30 \%)$ and Abraha's combination $(>40 \%)$ waveforms has been recorded. The potential of our approach has been examined considering a 1 Gbps OOK and 0.5 Gbps PPM modulation formats, a better bit error rate performance and higher power efficiencies have been achieved, while propagating over a large extension of single mode fiber. Adopting the chirping scheme with different optical amplifiers is a good way to extend our research, so as to make a comparison in terms of performance and system complexity.

\section{REFERENCES}

[1] I. Oppermann, M. Hämäläinen, and J. Iinatti, "UWB: theory and applications," John Wiley \& Sons, 2005.

[2] M. Ghavami, L. Michael, and R. Kohno, "Ultra wideband signals and systems in communication engineering," John Wiley \& Sons, 2007.

[3] G. R. Aiello and G. D. Rogerson, "Ultra-wideband wireless systems," IEEE Microwave Magazine, 4(2), pp. 36-47, 2003.

[4] FCC Report and Order. In the Matter of Revision of Part 15 of the Commission's Rules Regarding Ultra-Wideband Transmission Systems. FCC 02-48, Apr. 2002.

[5] M. Z. Win and R. A. Scholtz, "Impulse radio: How it works," IEEE Communications Lett., 2(2), pp. 36-38, 1998.

[6] H. Sheng, P. Orlik, A. M. Haimovich, Jr. L. J. Cimini, and J. Zhang, "On the spectral and power requirements for ultra-wideband transmission," In Communications, 2003. ICC'03. IEEE International Conference, Vol. 1, pp. 738-742, 2003.

[7] J. R. Fernandes and D. Wentzloff, "Recent advances in IR-UWB transceivers: An overview," In Circuits and Systems (ISCAS), Proceedings of 2010 IEEE International Symposium, pp. 3284-3287, 2010.

[8] C. Lim, A. Nirmalathas, M. Bakaul, P. Gamage, K. L. Lee, Y. Yang, R. Waterhouse, "Fiber-wireless networks and subsystem technologies," IEEE J. Lightw. Technol., 28(4), pp. 390-405, 2010.

[9] F. Zeng, and J. Yao, "An approach to ultrawideband pulse generation and distribution over optical fiber," IEEE Photon. Technol. Letters, 18(7), pp. 823-825, 2006.

[10] S. Pan, and J. Yao, "Performance evaluation of UWB signal transmission over optical fiber," IEEE J. Selected Areas in Communications, vol. 28, no.6, pp. 889-900, 2010.

[11] M. Popov, "The convergence of wired and wireless services delivery in access and home networks," In Optical Fiber Communication Conference (p. OWQ6). Optical Society of America, 2010.
[12] N. J. Gomes, M. Morant, A. Alphones, B. Cabon, J.E. Mitchell, C. Lethien, and S. Iezekiel, "Radio-over-fiber transport for the support of wireless broadband services," J. of Optical Networking, 8(2), pp. 156$178,2009$.

[13] F. Zeng, Q. Wang, and J. Yao, "All-optical UWB impulse generation based on cross-phase modulation and frequency discrimination," Electron. Lett., 43(2), pp. 119-121, 2007.

[14] M. Bolea, J. Mora, B. Ortega, and J. Capmany, "Optical UWB pulse generator using an $\mathrm{N}$ tap microwave photonic filter and phase inversion adaptable to different pulse modulation formats," Optics express, 17(7), pp. 5023-5032, 2009.

[15] Y. Yu, J. Dong, X. Li, and X. Zhang, "Ultra-wideband generation based on cascaded Mach-Zehnder modulators," IEEE Photon. Technol. Letters, 23(23), pp. 1754-1756, 2011.

[16] M. J. Connelly, Semiconductor Optical Amplifiers. Boston, MA:Kluwer, 2002.

[17] A. D. McCoy, P. Horak, B. C., Thomsen, M. Ibsen, \& D. J. Richardson, "Noise suppression of incoherent light using a gain-saturated SOA: Implications for spectrum-sliced WDM systems," Journal of Light wave Technology, 23(8), 2399, 2005.

[18] P. Baveja, D. N. Maywar, A. M. Kaplan, \& G. P. Agrawal, "Self-phase modulation in semiconductor optical amplifiers: impact of amplified spontaneous emission," IEEE Journal of Quantum Electronics, 46(9), 1396-1403, 2010.

[19] H. Taki, S. Azou, A. Hamie, A. Al Housseini, A. Alaeddine and A. Sharaiha, "Simple pre-distortion schemes for improving the power efficiency of SOA-based IR-UWB over fiber systems," Optics coтmиnications, 382, 225-231, 2016.

[20] K. Roberts, L. Chuandong, L. Strawczynski, M. O'sullivan, and I. Hardcastle, "Electronic precompensation of optical nonlinearity," IEEE Photon. Technol. Letters, 18(1-4), 403-405, 2006.

[21] E. Ip and J. M. Kahn, "Compensation of dispersion and nonlinear impairments using digital back propagation," J. Lightw. Technol., 26(20), 3416-3425, 2008.

[22] A. J. Lowery, "Fiber nonlinearity pre-and post-compensation for longhaul optical links using OFDM," Optics Express, 15(20), 12965-12970, 2007.

[23] J. Kim, \& K. Konstantinou, "Digital predistortion of wideband signals based on power amplifier model with memory," Electronics Letters, 37(23), 1, 2001.

[24] R. Marsalek, P. Jardin, \& G. Baudoin, "From post-distortion to predistortion for power amplifiers linearization," IEEE Communications Letters, 7(7), 308-310, 2003

[25] X. Zhang, R. Zhu, D. Shen, and T Liu, "Linearization Technologies for Broadband Radio-Over-Fiber Transmission Systems," Photonics, vol. 1, no.4, pp. 455-472, 2014.

[26] C. Tai, S.-L. Tzeng, H.-C. Chang, and W. I. Way, "Reduction of nonlinear distortion in mqw semiconductor optical amplifier using light injection and its application in multichannel m-qam signal transmission systems," IEEE Photon. Technol. Lett. , vol. 10, pp. 609-611, Apr. 1998

[27] F. Tabatabai, H. S. Al-Raweshidy, "Feed-Forward Linearization Technique for Reducing Non-Linearity in Semiconductor Optical Amplifier," J. Lightw. Technol., vol.25, No. 9, pp.2667-2674, Sep. 2007.

[28] S. Boll, "Suppression of acoustic noise in speech using spectral subtraction," IEEE Transactions on acoustics, speech, and signal processing, 27(2), 113-120, 1979

[29] B. Nikfal, Q. Zhang, \& C. Caloz, "Enhanced-SNR impulse radio transceiver based on phasers," IEEE Microwave and Wireless Components Letters, 24(11), 778-780, 2014.

[30] H. Khaleghi, P. Morel, A. Sharaiha, and T. Rampone, "Experimental validation of numerical simulations and performance analysis of a coherent optical-OFDM transmission system employing a semiconductor optical amplifier," J. Lightw. Technol., 31(1), pp. 161-170, 2013.

[31] S.T. Abraha, C. Okonkwo, P. A. Gamage, E. Tangdiongga, and T. Koonen, "Routing of power efficient IR-UWB wireless and wired services for in-building network applications," J. Lightw. Technol., 30(11), pp. 1651-1663, 2012.

[32] Q. Zhang, D. L. Sounas, \& C. Caloz, "Synthesis of cross-coupled reduced-order dispersive delay structures (DDSs) with arbitrary group delay and controlled magnitude," IEEE Transactions on Microwave Theory and Techniques, 61(3), 1043-1052, 2013.

[33] B. Nikfal, B., S. Gupta, \& C. Caloz, "Increased group-delay slope loop system for enhanced-resolution analog signal processing," IEEE 
Transactions on Microwave Theory and Techniques, 59(6), 1622-1628, 2011.

[34] B. Nikfal, D. Badiere, M. Repeta, B. Deforge, S. Gupta, \& C. Caloz, "Distortion-less real-time spectrum sniffing based on a stepped groupdelay phaser," IEEE microwave and wireless components letters, 22(11), 601-603, 2012.

[35] L. Cohen, Time-Frequency Analysis. Englewood Cliffs, NJ: PrenticeHall, 1995.

[36] H. Jin, L. Jiang, W. Hao, C. Sheng, H. Qijun, and Z. Yueping, "A CMOS fifth-derivative Gaussian pulse generator for UWB applications," Journal of Semiconductors, 35(9), 095005, 2014.

[37] R. Llorente, T. Alves, M. Morant, M. Beltran, J. Perez, A. Cartaxo, and J. Marti, "Ultra-wideband radio signals distribution in FTTH networks," IEEE Photon. Technol. Lett., 20(11), pp. 945-947, 2008. 